\title{
Cerebral Aneurysm Pulsation: Do Iterative Reconstruction Methods Improve Measurement Accuracy In Vivo?
}

\author{
T. Illies, D. Säring, M. Kinoshita, T. Fujinaka, M. Bester, J. Fiehler, N. Tomiyama, and Y. Watanabe
}

\begin{abstract}
BACKGROUND AND PURPOSE: Electrocardiogram-gated 4D-CTA is a promising technique allowing new insight into aneurysm pathophysiology and possibly improving risk prediction of cerebral aneurysms. Due to the extremely small pulsational excursions ( $<0.1 \mathrm{~mm}$ in diameter), exact segmentation of the aneurysms is of critical importance. In vitro examinations have shown improvement of the accuracy of vessel delineation by iterative reconstruction methods. We hypothesized that this improvement shows a measurable effect on aneurysm pulsations in vivo.
\end{abstract}

MATERIALS AND METHODS: Ten patients with cerebral aneurysms underwent 4D-CTA. Images were reconstructed with filtered backprojection and iterative reconstruction. The following parameters were compared between both groups: image noise, absolute aneurysm volumes, pulsatility, and sharpness of aneurysm edges.

RESULTS: In iterative reconstruction images, noise was significantly reduced (mean, $9.8 \pm 4.0$ Hounsfield units versus $8.0 \pm 2.5$ Hounsfield units; $P=.04$ ), but the sharpness of aneurysm edges just missed statistical significance (mean, $3.50 \pm 0.49 \mathrm{~mm}$ versus $3.42 \pm 0.49 \mathrm{~mm} ; P=.06$ ). Absolute volumes (mean, $456.1 \pm 775.2 \mathrm{~mm}^{3}$ versus $461.7 \pm 789.9 \mathrm{~mm}^{3} ; P=.31$ ) and pulsatility (mean, $1.099 \pm 0.088 \mathrm{~mm}^{3}$ versus $1.095 \pm 0.082 \mathrm{~mm}^{3} ; P=.62$ ) did not show a significant difference between iterative reconstruction and filtered back-projection images.

CONCLUSIONS: CT images reconstructed with iterative reconstruction methods show a tendency toward shorter vessel edges but do not affect absolute aneurysm volumes or pulsatility measurements in vivo.

ABBREVIATIONS: AIDR = adaptive iterative dose reduction; IR = iterative reconstruction; FBP $=$ filtered back-projection; $\mathrm{HU}=\mathrm{Hounsfield}$ units

E lectrocardiogram-gated 4D-CT angiography has been used to -analyze the pulsation of cerebral aneurysms. ${ }^{1-7}$ Insight into aneurysm pathophysiology and improvement of risk prediction of incidental cerebral aneurysms can be expected. The technique is limited by the small pulsational excursions of cerebral aneurysms. If one considers a volume change of $5 \%$ within the cardiac cycle, the change in diameter of a spherical aneurysm of $5 \mathrm{~mm}$ diameter is on the order of $0.1 \mathrm{~mm},{ }^{1,2}$ which is below the resolution of CTA. Exact segmentation of the aneurysm is, therefore, critical and of the utmost importance for the correct analysis of pulsations.

Received January 21, 2014; accepted after revision April 14.

From the Departments of Diagnostic and Interventional Neuroradiology (T.I., M.B., J.F.) and Medical Informatics (D.S.), University Medical Center Hamburg-Eppendorf, Hamburg, Germany; and Departments of Neurosurgery (M.K., T.F.) and Radiology (N.T., Y.W.), Osaka University Graduate School of Medicine, Osaka, Japan.

This work was supported by a grant of the Japanese German Radiology Affiliation. Please address correspondence to Till Illies, MD, Department of Diagnostic and Interventional Neuroradiology, University Medical Center Hamburg-Eppendorf, Martinistr 52, 20355 Hamburg, Germany; e-mail: tillies@uke.de

http://dx.doi.org/10.3174/ajnr.A4000
In vitro experiments with vascular models have shown that vessel delineation depends on various factors, including intraluminal contrast attenuation, vascular wall thickness, postprocessing, and reconstruction methods. ${ }^{8}$ Iterative reconstruction (IR) algorithms have gained importance in clinical routine CT because the radiation dose can be reduced significantly while image quality is maintained compared with filtered back-projection (FBP) reconstruction. At a constant radiation dose, IR reduces image blur, enhances edges, and increases image resolution. ${ }^{8-10}$ Depiction of vessels in the posterior fossa and the spinal canal ${ }^{11,12}$ is improved in vivo. Moreover, in vitro studies reveal improvement of the accuracy of quantitative measurement of vessel diameters. ${ }^{8}$

These findings and its overall characteristics make IR an interesting tool for improving the accuracy of pulsation measurements of cerebral aneurysms. To our knowledge, the influence of IR on vessel-volume measurement, especially in $4 \mathrm{D}-\mathrm{CTA}$, has not been examined in vivo. We hypothesized that IR methods have a measureable effect on the accuracy of quantification of cerebral aneurysm pulsation in vivo. 


\section{MATERIALS AND METHODS \\ Patients}

Ten patients with unruptured cerebral aneurysms underwent 4DCTA. Four aneurysms were located at the internal carotid artery; 4 , at the middle cerebral artery; 1 , at the anterior communicating artery; and 1, at the anterior cerebral artery. The local ethics committee approved the use of the clinical data for research and waived the requirement for written informed consent from patients. Patient data are listed in the Table.

\section{D-CTA Acquisition}

Retrospectively electrocardiogram-gated CTA was performed on a 320-detector row Aquilion ONE CT scanner (Toshiba Medical Systems, Tokyo, Japan). We used the following parameters: $120-\mathrm{kV}$ tube voltage, 270-mA tube current, 350-ms gantry rotation time, 140-mm z-coverage. Contrast medium (ioversol, Optiray $320 \mathrm{mg} \mathrm{I/mL;} \mathrm{Covidien} \mathrm{Japan,} \mathrm{Tokyo,} \mathrm{Japan)} \mathrm{was} \mathrm{injected} \mathrm{at}$ $5 \mathrm{~mL} / \mathrm{s}$. Timing for the image aquisition was determined with a test injection of $15 \mathrm{~mL}$ of contrast medium. For CTA, $50 \mathrm{~mL}$ of contrast medium was injected followed by a saline flush. 4DCTAs were reconstructed with half reconstruction by using FBP and a kernel optimized for intracranial vessel imaging and IR

\section{Patient characteristics}

\begin{tabular}{|c|c|c|c|c|c|}
\hline \multirow{2}{*}{$\begin{array}{c}\text { Patient } \\
\text { No. }\end{array}$} & \multirow{2}{*}{$\begin{array}{l}\text { Age } \\
\text { (yr) }\end{array}$} & \multirow{2}{*}{$\begin{array}{l}\text { Location } \\
\text { (Artery) }\end{array}$} & \multicolumn{2}{|c|}{ Volume $(\mathrm{mm} 3)^{\mathrm{a}}$} & \multirow{2}{*}{$\begin{array}{c}\text { Diameter } \\
(\mathrm{mm})^{\mathrm{b}}\end{array}$} \\
\hline & & & AIDR & FBP & \\
\hline 1 & 53 & MCA & 2336.4 & 2386.0 & 16.7 \\
\hline 2 & 66 & AcomA & 95.1 & 95.7 & 6.1 \\
\hline 3 & 55 & ICA & 69.1 & 72.9 & 5.7 \\
\hline 4 & 70 & MCA & 15.3 & 15.4 & 3.9 \\
\hline 5 & 50 & ICA & 1352.5 & 1361.7 & 15.1 \\
\hline 6 & 69 & $A C A$ & 67.4 & 67.3 & 4.3 \\
\hline 7 & 61 & ICA & 76.7 & 77.2 & 5.9 \\
\hline 8 & 76 & MCA & 58.6 & 59.2 & 5.7 \\
\hline 9 & 50 & ICA & 408.8 & 407.1 & 10.7 \\
\hline 10 & 68 & MCA & 82.2 & 74.4 & 7.1 \\
\hline
\end{tabular}

Note:-AcomA indicates anterior communicating artery; ACA, anterior cerebral artery.

${ }^{a}$ For volume measurement, a 3D model of the vasculature was created and the aneurysms were interactively segmented (see "Material and Methods" section).

$\mathrm{b}$ The aneurysm diameter is the maximum value of the axial, coronal, or sagittal aneurysm diameter. algorithm (adaptive iterative dose reduction [AIDR] 3D) with 10 steps of each $10 \%$ of the R-R interval, $512 \times 512$ image matrix, $0.5-\mathrm{mm}$ section thickness, and $0.39 \times 0.39 \mathrm{~mm}$ in-plane resolution.

\section{Postprocessing}

We used an in-house-developed software as well as ImageJ software (National Institutes of Health, Bethesda, Maryland) and MeVisLab (MeVis Medical Solutions, Bremen, Germany) for volume measurement, and R (http://www.r-project.org/) and R Studio 0.97 (http://rstudio.org/download/desktop) for statistical analysis.

\section{Noise}

Mean and SDs were calculated from circular ROIs in air (400 $\left.\mathrm{mm}^{2}\right)$, brain tissue $\left(200 \mathrm{~mm}^{2}\right)$, and the aneurysm on transversal sections in all phases in FBP and AIDR images. The SDs of the densities within the ROIs were averaged over all timeframes, and their means and SDs were compared between the FBP and AIDR images with $t$ tests as a measure of objective image noise.

\section{Edge Sharpness}

For each aneurysm, 1 attenuation profile along a line passing through its center was acquired on axial images. Measurement points were interpolated by using cubic splines. The width of the edge response of the aneurysm wall was defined as the distance between points corresponding to $10 \%$ and $90 \%$ of the maximum intra-aneurysmal attenuation on the border of the aneurysms. A shorter edge response corresponds to a sharper edge representation. Edge responses were compared between FBP and IR groups (Fig 1).

\section{Absolute Volumes and Pulsatility}

Datasets were loaded into an inhouse-developed postprocessing software. Perivascular tissue was removed with a thresholded segmentation (160-
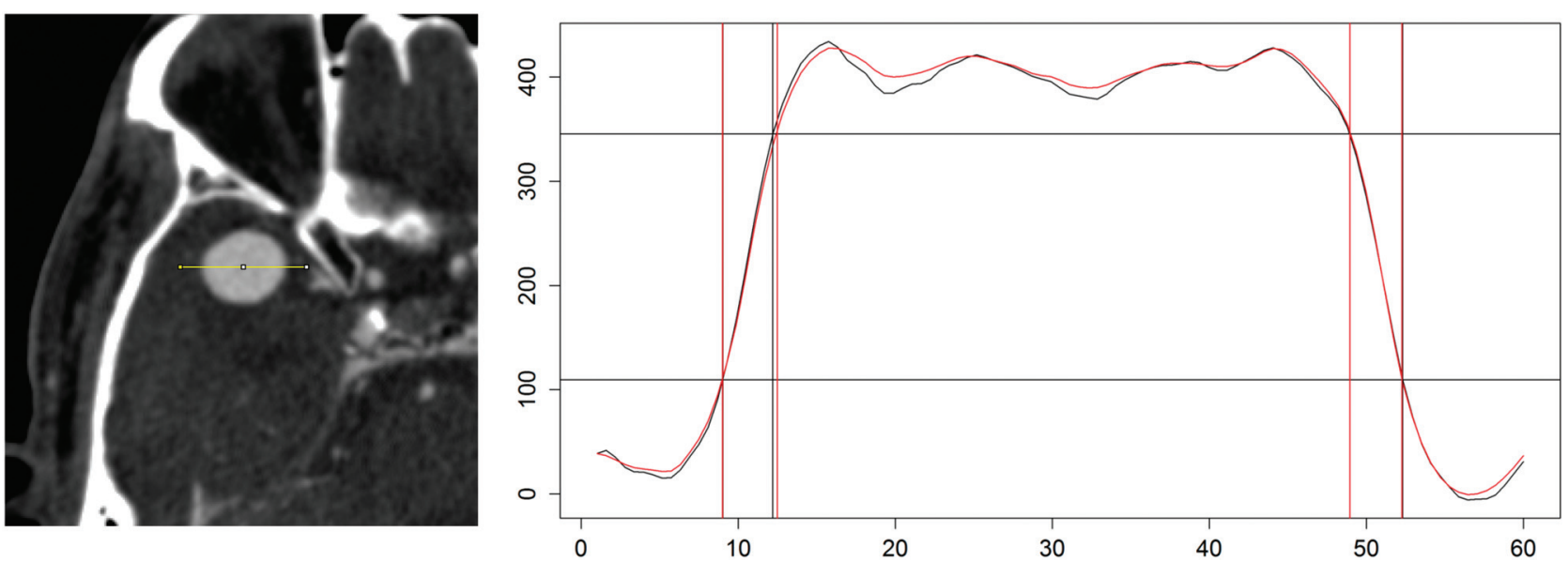

FIG 1. Creation of a line profile across the aneurysms for calculations of edge lengths. The left side shows a right middle cerebral artery aneurysm. A linear region of interest is positioned through the center of the aneurysm. The right side shows a cubic spline function fitted to the measurements for FBP (black) and AIDR (red). Edges are defined as the distance between the $10 \%$ and $90 \%$ interval of the maximum intraaneurysmal attenuation (horizontal black lines). 

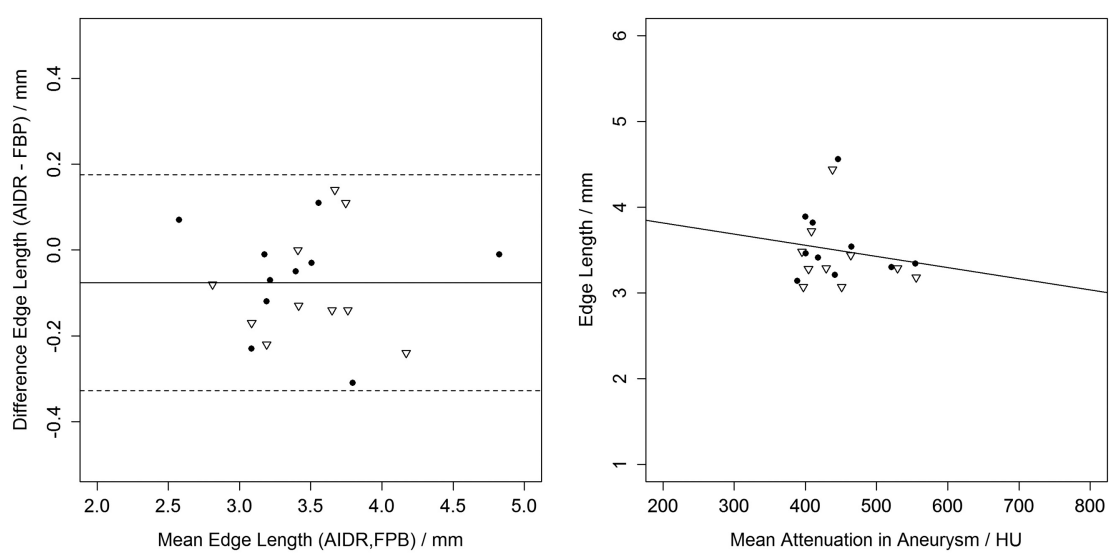

FIG 2. Edge lengths compared between AIDR and FBP and edge length compared with intraaneurysmal attenuation (triangles, left side; dots, right side). On the left side, a Bland-Altman plot shows a small bias of shorter edges in AIDR images. Th right side shows the plot and regression line of edge length and intra-aneurysmal attenuation $(P=.45)$.
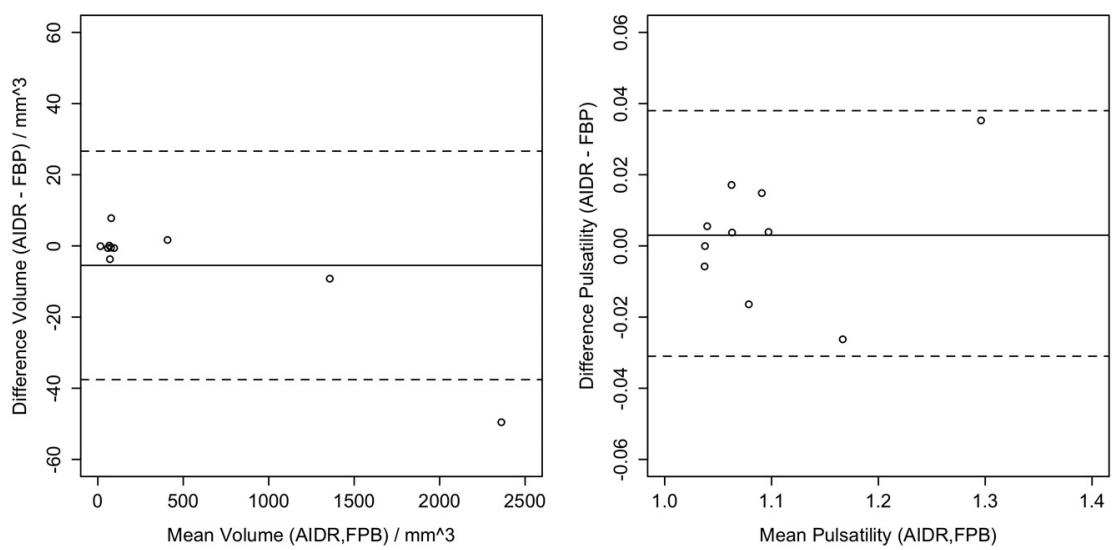

FIG 3. Bland-Altman plots of absolute volumes and pulsatility. Left side shows a small bias, with a tendency toward smaller absolute volumes in AIDR images. On the right side, there are no differences in pulsatility between the aneurysms in AIDR and FBP images.
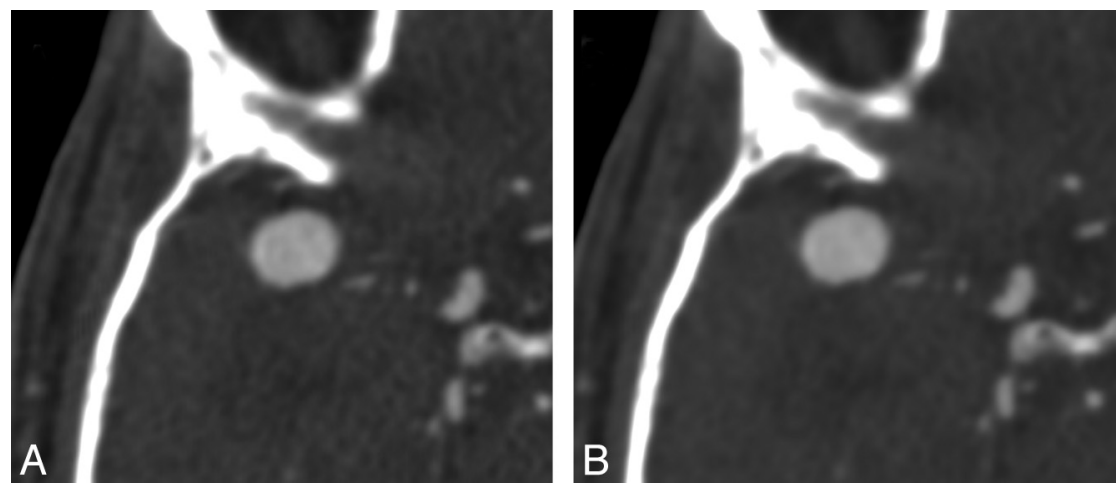

FIG 4. Aneurysm of the right middle cerebral artery in a 53-year-old patient (patient 1). CT angiography of the 2 reconstruction methods; acquisition parameters are the same. $A$, Filtered back-projection. $B$, Image reconstruction with adaptive iterative dose reduction. Note the smoother appearance of the image, consistent with reduced image noise. time point zero. This initial cutting line was transferred automatically to all other time points by using an iterative closestpoint approach, to reduce interaction time. Now, the surface model of the aneurysm for each time point was generated on the basis of the transferred cutting lines. For analysis, the absolute volumes and maximum and minimum volumes of the aneurysm surface model within the cardiac cycle were calculated and compared between both groups. Pulsatility was calculated as the ratio of maximum and minimum volumes and was compared between both groups.

\section{RESULTS}

\section{Noise}

In ROIs placed in the air, mean intensity values were $-1040.2 \pm 22.2 \mathrm{HU}$ versus $-1040.6 \pm 22.1 \mathrm{HU}(P=.97)$ and SDs were $9.8 \pm 4.0 \mathrm{HU}$ versus $8.0 \pm 2.5 \mathrm{HU}$ $(P=.04)$ (FBP versus AIDR). In brain parenchyma, the means were $53.7 \pm 5.0$ HU versus $53.9 \pm 4.8 \mathrm{HU}(P=.91)$ and the SDs were $15.0 \pm 3.5 \mathrm{HU}$ versus $11.9 \pm 1.9 \mathrm{HU}(P=.03)$ (FBP versus AIDR); and in aneurysms, the means were $433.0 \pm 136.1 \mathrm{HU}$ versus $427.0 \pm$ 132.6 HU $(P=.89)$ and the SDs were $20.3 \pm 7.4 \mathrm{HU}$ versus $14.6 \pm 5.2 \mathrm{HU}$ $(P<.001)$ (FBP versus AIDR).

\section{Edge Sharpness}

Mean edge length was $3.50 \pm 0.49 \mathrm{~mm}$ versus $3.42 \pm 0.49 \mathrm{~mm}$ (FBP versus AIDR). Bland-Altmann analysis revealed a bias of $-0.08 \mathrm{~mm}$ (AIDR-FBP, $P=.06$, paired $t$ test) and limits of agreement between -0.32 and $0.19 \mathrm{~mm}$. Edge sharpness did not correlate significantly with intraluminal attenuation $(P=.45$, $\left.r^{2}=0.032\right)($ Fig 2).

\section{Absolute Volumes}

Mean aneurysm size was $461.7 \pm 789.9$ $\mathrm{mm}^{3}$ versus $456.1 \pm 775.2 \mathrm{~mm}^{3}$ (FBP versus AIDR). Bland-Altman analysis revealed a bias of $-5.6 \mathrm{~mm}^{3}$ (AIDRFBP, $P=.31$, paired $t$ test) and limits of agreement of -37.6 and $26.6 \mathrm{~mm}^{3}$ for mean volumes (Fig 3).
890 Hounsfield units [HU]). On the basis of the segmentation of the aneurysm and the attached vessel system, a single surface model was generated by using the marching cube algorithm. To separate the aneurysm structure from the vessels, the observer manually selected a number of points representing the cutting line at the

\section{Pulsatility}

Mean pulsatility was $1.095 \pm 0.082 \mathrm{~mm}^{3}$ versus $1.099 \pm 0.088 \mathrm{~mm}^{3}$ (FBP versus AIDR), and Bland-Altman analysis revealed a bias of 0.003 (AIDR-FBP, $P=.62$, paired $t$ test), with limits of agreement between -0.03 and 0.04 (Fig 4). 


\section{DISCUSSION}

Analysis of the pulsations of cerebral aneurysms could improve the understanding of mechanisms involved in their enlargement and rupture and increase the accuracy of risk prediction of unruptured aneurysms. Previous studies found volume changes of cerebral aneurysms within the cardiac cycle of approximately 5\%. ${ }^{1}$ These pulsations did not differ significantly from those of normal cerebral arteries but showed a tendency toward larger values. Due to the small excursions of the aneurysm wall, pulsation analysis is technically challenging and improving its accuracy is demanding. IR methods have been shown to improve measurement precision of vessel diameter in a phantom study, a finding from which aneurysm pulsation analysis could benefit. We compared pulsations of 10 cerebral aneurysms from FBP and AIDR reconstructed datasets to analyze the effect size in vivo. No significant changes were observed in absolute aneurysm volumes and pulsations between images reconstructed with IR and AIDR. However, vessel sharpness, one of AIDR's characteristics for improving measurement accuracy, was increased, a finding that just missed statistical significance.

The aforementioned phantom study investigated the influence of wall thickness, intraluminal attenuation, and the reconstruction method on diameter measurement. In our study, the wall thicknesses of aneurysms were essentially unknown but could be estimated by the results of previous reports. In a pathologically based study, the wall thickness was found to be $0.25 \mathrm{~mm}$ for $10-\mathrm{mm}^{13}$ aneurysms, and an MR imaging-based study found a wall size of $0.6 \mathrm{~mm} .{ }^{14}$ Mean intra-aneurysmal attenuation was $430 \mathrm{HU}$, ranging from 223 to 555 HU for the aneurysms, due to the capture of the bolus in first-pass $4 \mathrm{D}$-CTA. Of the 9 groups in the phantom study, for the one comparable to our samples with respect to wall thickness $(0.5 \mathrm{~mm})$ and intraluminal attenuation (396 HU), a difference in diameter measurement between FBP and IR images could not be proved. While vessel diameters were overestimated with both reconstruction methods, IR reduced measurement errors significantly in larger-diameter vessels and with higher intraluminal attenuation. Our findings in humans reflect these results by showing smaller volumes in all aneurysms in the AIDR group. However, this finding did not reach a statistically significant level. For a small effect size as found in our study, a much larger sample size is needed to achieve sufficient statistical power.

The effect of IR on vessel delineation is attributed to its ability to improve resolution, reduce image noise, and enhance edges. ${ }^{15}$ The examination of image resolution with respect to the reconstruction method is beyond the scope of this article and has been shown in previous examinations. ${ }^{16}$ AIDR-reconstructed images expectedly showed reduced overall noise levels, and intra-aneurysmal noise was decreased by $28 \%$ (Fig 4 ). The ability of IR to reduce noise and enhance edges at the same time distinguishes it from FBP reconstruction, which compromises between these as it uses a filter kernel. A quantitative measure for edge sharpness is the length of the attenuation profile at the vessel border, ranging from $10 \%$ to $90 \%$ of the maximum intraluminal attenuation. The previously described phantom study also examined the influence of wall thickness, intraluminal attenuation, and reconstruction methods on this edge sharpness. IR images showed shorter edges throughout all groups. Edge lengths in our study just missed a statistically significant level but showed a tendency toward shorter values in AIDR-reconstructed images as seen in the phantom study (Fig 2). There, vessels with 0.5 -mm walls did not show an edge profile corresponding to the actual vessel wall as was seen in the thicker-walled vessels. The lack of depiction of the wall in thin-walled vessels was attributed to reconstruction blur and might be the major source of the lack of improvement of accuracy in measuring vessel diameters in this group. With an estimated wall thickness of $0.25 \mathrm{~mm}$ in aneurysms in vivo, this effect is even more pronounced.

Due to the small sample size, our study lacks the statistical power to draw further conclusions. Hence, the absence of a significantly different absolute volume or pulsation fraction does not translate into a lack of improvement of accuracy by IR in pulsation analysis. The effect size is small, necessitating large study groups. However, further effort should be made because our results show the applicability of the findings of a well-controlled in vitro study that proved the superiority of IR methods in the diameter measurement of vessels. Furthermore, IR methods from different vendors may have variable effects on edge enhancement and could show a larger improvement of accuracy for this specific application.

\section{CONCLUSIONS}

IR methods do not increase the accuracy of pulsation measurements of cerebral artery aneurysms in vivo. This finding might be mainly due to their small wall size.

Disclosures: Till Illies—RELATED: Support for Travel to Meetings for the Study or Other Purposes: Japanese German Radiology Affiliation, Comments: for an exchange to Osaka, Japan. Jens Fiehler-UNRELATED: Consultancy: Codman, Stryker, MicroVention; Grants/Grants Pending: Covidien, Stryker, MicroVention*; Payment for Lectures Including Service on Speakers Bureaus: Codman, Covidien, Stryker, MicroVention, Penumbra; Travel/Accommodations/Meeting Expenses Unrelated to Activities Listed: Covidien. Yoshiyuki Watanabe-RELATED: Grant: Toshiba Medical, ${ }^{*}$ Comments: research fund about radiation dose reduction using 320-detector row CT. *Money paid to the institution.

\section{REFERENCES}

1. Kuroda J, Kinoshita M, Tanaka H, et al. Cardiac cycle-related volume change in unruptured cerebral aneurysms: a detailed volume quantification study using 4-dimensional CT angiography. Stroke 2012;43:61-66

2. Umeda Y, Ishida F, Hamada K, et al. Novel dynamic four-dimensional CT angiography revealing 2-type motions of cerebral arteries. Stroke 2011;42:815-18

3. Yaghmai V, Rohany M, Shaibani A, et al. Pulsatility imaging of saccular aneurysm model by 64-slice CT with dynamic multiscan technique. J Vasc Interv Radiol 2007;18:785-88

4. Matsumoto M, Sasaki T, Suzuki K, et al. Visualizing the dynamics of cerebral aneurysms with four-dimensional computed tomographic angiography. Neurosurgery 2006;58:E1003, author reply E1003

5. Kato $\mathrm{Y}$, Hayakawa $M$, Sano $\mathrm{H}$, et al. Prediction of impending rupture in aneurysms using 4D-CTA: histopathological verification of a real-time minimally invasive tool in unruptured aneurysms. Minim Invasive Neurosurg 2004;47:131-35

6. Ishida F, Ogawa H, Simizu T, et al. Visualizing the dynamics of cerebral aneurysms with four-dimensional computed tomographic angiography. Neurosurgery 2005;57:460-71, discussion 460-71

7. Hayakawa M, Katada K, Anno H, et al. CT angiography with electrocardiographically gated reconstruction for visualizing pulsation of intracranial aneurysms: identification of aneurysmal protuberance presumably associated with wall thinning. AJNR Am J Neuroradiol 2005;26:1366-69 
8. Suzuki S, Machida, H, Tanaka I, et al. Vascular diameter measurement in CT angiography: comparison of model-based iterative reconstruction and standard filtered back projection algorithms in vitro. AJR Am J Roentgenol 2013;200:652-57

9. Ziegler A, Köhler T, Proksa R. Noise and resolution in images reconstructed with FBP and OSC algorithms for CT. Med Phys 2007; 34:585-98

10. Sprawls P. AAPM tutorial: CT image detail and noise. Radiographics 1992;12:1041-46

11. Machida H, Takeuchi H, Tanaka I, et al. Improved delineation of arteries in the posterior fossa of the brain by model-based iterative reconstruction in volume-rendered 3D CT angiography. AJNR Am J Neuroradiol 2013;34:971-75

12. Machida H, Tanaka I, Fukui R, et al. Improved delineation of the anterior spinal artery with model-based iterative reconstruction in CT angiography: a clinical pilot study. AJR Am J Roentgenol 2013; 200:442-46

13. Steiger HJ, Aaslid R, Keller S, et al. Strength, elasticity and viscoelastic properties of cerebral aneurysms. Heart Vessels 1989;5:41-46

14. Park JK, Lee CS, Sim KB, et al. Imaging of the walls of saccular cerebral aneurysms with double inversion recovery black-blood sequence. J Magn Reson Imaging 2009;30:1179-83

15. Beister M, Kolditz D, Kalender WA. Iterative reconstruction methods in X-ray CT. Phys Med 2012;28:94-108

16. Suzuki S, Machida H, Tanaka I, et al. Measurement of vascular wall attenuation: comparison of CT angiography using model-based iterative reconstruction with standard filtered back-projection algorithm CT in vitro. Eur J Radiol 2012;81:3348-53 